Check for updates

Cite this: RSC Adv., 2017, 7, 40510

Received 25th July 2017

Accepted 13th August 2017

DOI: 10.1039/c7ra08196k

rsc.li/rsc-advances

\section{Exploring sesquiterpene alkaloid-like scaffolds via Beckmann-transannular remodelling of beta-caryophyllene $\uparrow$}

\author{
Shuang-Jiang Ma, ${ }^{a}$ Jie Yu, (D) a Hua-Fang Fan, ${ }^{a}$ Zi-Han Li, ${ }^{c}$ An-Ling Zhang *a \\ and Qiang Zhang (D) *ab
}

\begin{abstract}
High-throughput screening (HTS) is the dominant approach to identify lead compounds in drug development. However, most compound-screening collections provide little structural or stereochemical complexity, which do not offer enough diversity to merit the modulation of many drug targets. Here we describe a facile strategy for the creation of diverse compounds with high structural and stereochemical complexity using readily available natural products, $\beta$-caryophyllene, as a synthetic starting point. Our findings demonstrate that a cascaded Beckmann-transannular protocol transforms macrocyclic natural products into poly-heterocyclic unnatural skeletal types. These compounds are significantly more complex and diverse than those in standard screening collections.
\end{abstract}

\section{Introduction}

Natural products (NPs) have played an essential role in developing novel drugs due to their structural diversity. ${ }^{1-4}$ Nevertheless, pharmaceutical research into NPs has recently declined owing to factors such as the difficulty of collecting novel compounds containing privileged structures. ${ }^{2}$ Thus, new strategies to augmenting chemical diversity are crucial for retaining the utility of natural products and their derivatives. Recently, creating complex small molecules directly from NPs has appeared as a convenience approach in diversity-oriented synthesis (DOS) area. $^{5-9}$ NPs are being considered as exceptional starting points due to the high abundance and structural variation. Additionally, NPs provide an excellent framework for site selective and stereoselective transformations.

The construction of heterocyclic molecules with potentially interesting biological activities represents an important part of the drug discovery process. Especially there has been a growing interest in the synthesis of medium-sized rings because of their many applications. ${ }^{10}$ Among them, poly-N-heterocyclic NPs displayed excellent bioactivities, such as homoharringtonine (topoisomerase inhibition, $\quad \mathrm{IC}_{50} \quad 9.0 \mathrm{nM}$ ), ${ }^{11}$ stenine $\quad$ B

${ }^{a}$ Shaanxi Key Laboratory of Natural Products \& Chemical Biology, College of Chemistry \& Pharmacy, Northwest A\&F University, Yangling 712100, PR China. E-mail: zhangq@nwsuaf.edu.cn; anlingzh@nwsuaf.edu.cn

${ }^{b}$ State Key Laboratory of Medicinal Chemical Biology, Nankai University, Tianjin 300071, PR China

${ }^{c}$ College of Life Sciences, Northwest A\&F University, Yangling 712100, PR China

$\dagger$ Electronic supplementary information (ESI) available: Experimental, characterization data, X-ray structures of compounds $\mathbf{6}$ and $\mathbf{9}$ and NMR spectra. CCDC 1552864 and 1519447. For ESI and crystallographic data in CIF or other electronic format see DOI: $10.1039 / \mathrm{c} 7 \mathrm{ra} 08196 \mathrm{k}$ (acetylcholinesterase inhibition $\mathrm{IC}_{50} 2.1 \mu \mathrm{M}$ ), ${ }^{12}$ sessilistemonamine $\mathrm{E}$ (acetylcholinesterase inhibition $\mathrm{IC}_{50} 9.1 \mu \mathrm{M}$ ), ${ }^{13}$ and cryptopleurin ( $\left.\mathrm{NF} \kappa_{\mathrm{B}}, \mathrm{IC}_{50} 1.4 \mathrm{nM}\right)^{14}$ (Fig. 1). Compounds of this type have been reported to show a wide range of potential applications in antitumor, anti-inflammatory and Alzheimer's disease therapy.

The propensity of macrocyclic precursors to undergo transannular cyclization has been deftly harnessed by nature to build
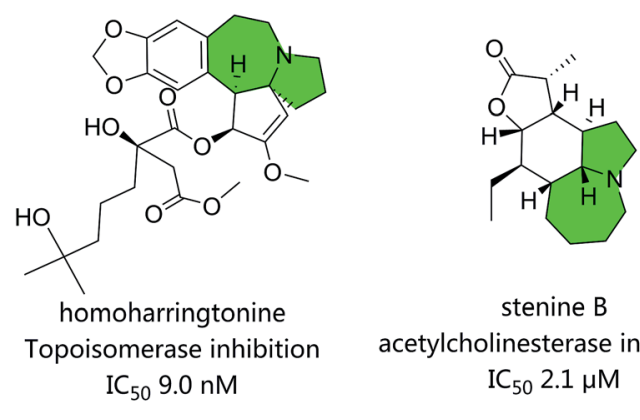

stenine $B$ acetylcholinesterase inhibition

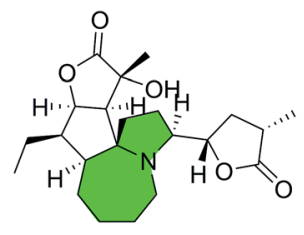

sessilistemonamine $\mathrm{E}$ acetylcholinesterase inhibition $\mathrm{IC}_{50} 9.1 \mu \mathrm{M}$

Fig. 1 Bioactive natural products with $\mathrm{N}$-containing poly-heterocyclic skeleton. 


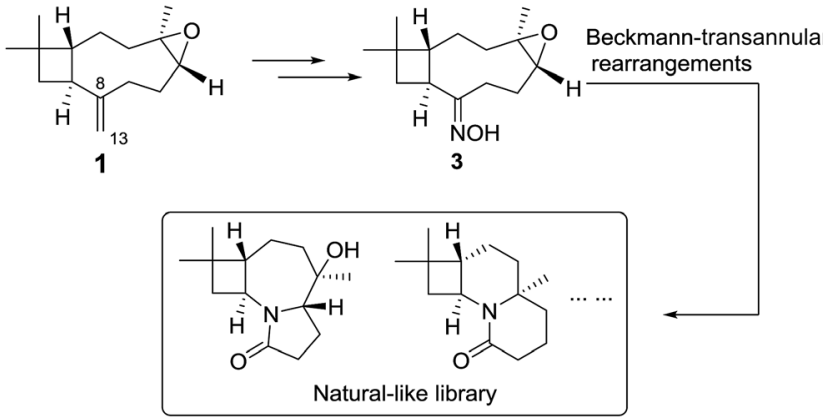

Scheme 1 Beckmann-transannular strategy to NP-like library.

thousands of polycyclic terpenoids and alkaloids with mediumsized rings. ${ }^{9,15-17}$ Our previous diversity-oriented transformation demonstrated the convenience protocol to build natural-like libraries containing medium-sized rings from macrocyclic caryophyllene. ${ }^{18,19}$ In this paper, we consider applying Beckmann rearrangement associated with transannular cyclization to explore chemical space covering poly-N-heterocyclic skeletons.

The natural sesquiterpenes, such as $\beta$-caryophyllene and its epoxide (1), are readily abundant to be ideal starting points to build diverse natural-like scaffolds along NP-driven DOS strategy. Due to the tensional cyclobutane, macrocyclic moiety and exocyclic olefin, compound $\mathbf{1}$ plays as a key precursor in nature to form diverse tricyclic sesquiterpenes by transannular rearrangements. ${ }^{20}$ In this paper, we reported a concise procedure to build natural-like skeletons with N-containing polyheterocyclic skeletons via Beckmann-transannular rearrangement on the macrocycle of caryophyllene (Scheme 1). To start Beckmann rearrangements on the macrocycle, compound $\mathbf{1}$ was introduced oxime group at C-8 as starting site (Scheme 1).

\section{Results and discussion}

The end olefin at C-8 in $\mathbf{1}$ was cleaved by $\mathrm{RuCl}_{3} / \mathrm{NaIO}_{4}$ oxidation to yield a 13-nor product (2) with carbonyl function group. The key intermediate oxime derivative (3) was prepared via condensation with hydroxylamine. In the next step, Lewis acid $\mathrm{LiBF}_{4} / \mathrm{MeCN}$ inspired 3 into a DOS library containing five compounds 4-8 (Scheme 2).
The structures of compounds 4-8 were unambiguously elucidated using 1D and 2D NMR and MS spectra. As a representative example, the structural analysis of $\mathbf{6}$ was elucidated in detail herein. Its molecular formula (MF) was assigned as $\mathrm{C}_{16} \mathrm{H}_{26} \mathrm{~N}_{2} \mathrm{O}_{2}$ by HRESIMS, indicating 5 degrees of unsaturation. Its ${ }^{13} \mathrm{C}$ and DEPT NMR spectroscopic data revealed the presence of two imine groups $\left(\delta_{\mathrm{C}} 161.1,162.4\right)$ and other 15 aliphatic carbons, including three methyls, five methenes, three methines and two quaternary carbon atoms. Apart from those two degrees of unsaturated $\mathrm{C}=\mathrm{N}$ bonds, the remaining implied that 6 possesses three rings in the structure.

Extensive analysis of the HSQC, ${ }^{1} \mathrm{H}-{ }^{1} \mathrm{H}$ COSY, and HMBC correlations (Table 1) established the whole planar structure. ${ }^{1} \mathrm{H}-{ }^{1} \mathrm{H}$ COSY correlations disclosed two isolated spin systems. HMBC correlations from $\mathrm{H}_{3}-15$ to $\mathrm{C}-1, \mathrm{C}-10, \mathrm{C}-11$ and $\mathrm{C}-14$ indicated a moiety of cyclobutane with a gem-dimethyl group. The HMBC correlations from $\mathrm{H}_{2}-10$ and $\mathrm{H}_{2}-7$ to C-8 indicated that the C-7 and C-9 are linked up at C-8. The HMBC correlations from $\mathrm{H}_{3}-12$ to $\mathrm{C}-3, \mathrm{C}-4$ and $\mathrm{C}-5$ disclosed a 9 membered ring. The dihydrooxazole ring with a methyl was identified by the HMBC correlations of $\mathrm{H}-5 / \mathrm{C}-16$ and $\mathrm{H}_{3}-17 / \mathrm{C}-16$. The relative configuration were analysed on the basis of NOESY correlations (Table 1). The NOE correlation of $\mathrm{H}-1 / \mathrm{H}-5$ indicated these $\mathrm{H}$ atoms are in $\beta$-orientation. The NOE correlations of $\mathrm{H}-9 / \mathrm{H}_{\alpha-7 /}$ $\mathrm{H}_{3}-12$ indicated the $\mathrm{H}-9$ and $\mathrm{H}_{3} \mathrm{C}-12$ are in $\alpha$-orientation. The relative configuration were also confirmed by $\mathrm{Cu} \mathrm{K} \alpha$ X-ray diffraction analysis (Table 1). However, high values of Flack parameters (0.03) indicated that the X-ray data did not provide unambiguous information about absolute configuration. Alternatively, based on the unchanged $(1 R)$ and $(9 S)$ configurations, the absolute configuration was deduced as therein on the basis of NOESY correlations related to $\mathrm{H}-1$ and $\mathrm{H}-9$.

Compound 6 possesses a 2-oxazoline moiety which was generated from epoxyethane moiety in 3 and the solvent acetonitrile. The 2-oxazoline ring was reported to be synthesized in good yields catalyst by $\mathrm{BF}_{3}-\mathrm{OEt}_{2}{ }^{21}$ or $\mathrm{SiF}_{4} \cdot{ }^{22}$ In the current reaction, $\mathrm{LiBF}_{4}$ was considered to decompose into $\mathrm{BF}_{3}$ and LiF. Compound $\mathbf{3}$ was transformed into carbonium ion $\mathbf{A}$ catalysed by $\mathrm{BF}_{3}$ (Scheme 2). The intermediate $\mathbf{A}$ was attacked by $\mathrm{F}^{-}$or MeCN to yield fluoride 4 and 2-oxazoline derivate $\mathbf{6}$. Simultaneously, elimination reactions of $\mathbf{A}$ afforded products 5, 7 and 8 .

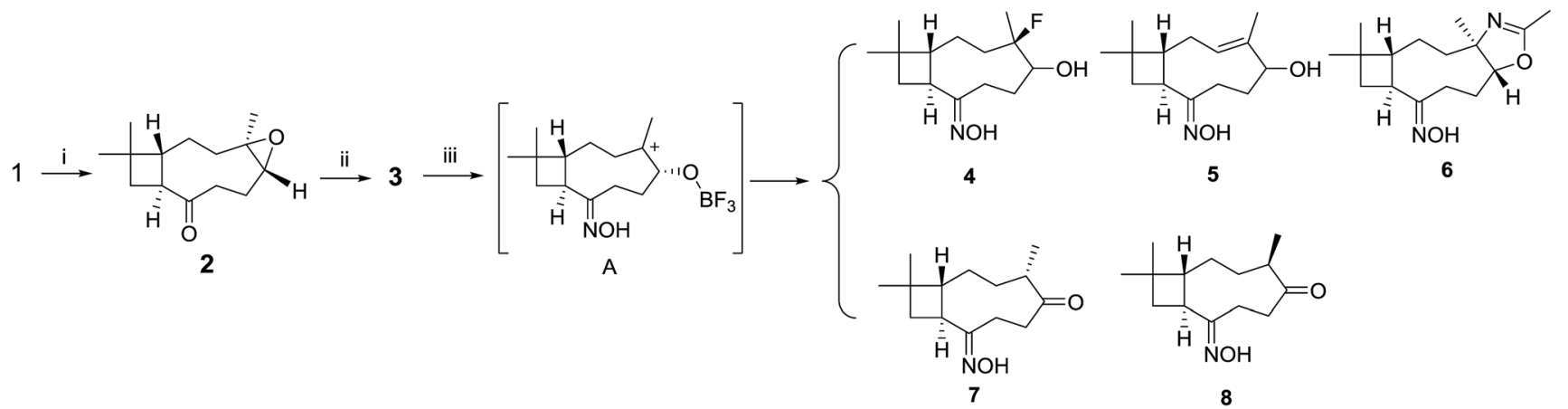

Scheme 2 Diversity-oriented transformation to oxime. Reaction condition: (i) $\mathrm{NalO}_{4}, \mathrm{RuCl}_{3}, \mathrm{MeCN}-\mathrm{EtOAc}-\mathrm{H}_{2} \mathrm{O}$; (ii) $\mathrm{NH} \mathrm{H}_{2} \mathrm{OH} \cdot \mathrm{HCl}, \mathrm{NaOH}$; (iii) $\mathrm{LiBF}_{4}, \mathrm{MeCN}, 45^{\circ} \mathrm{C}$. 
Table 1 Structural analysis of compounds 6 and $9^{a}$

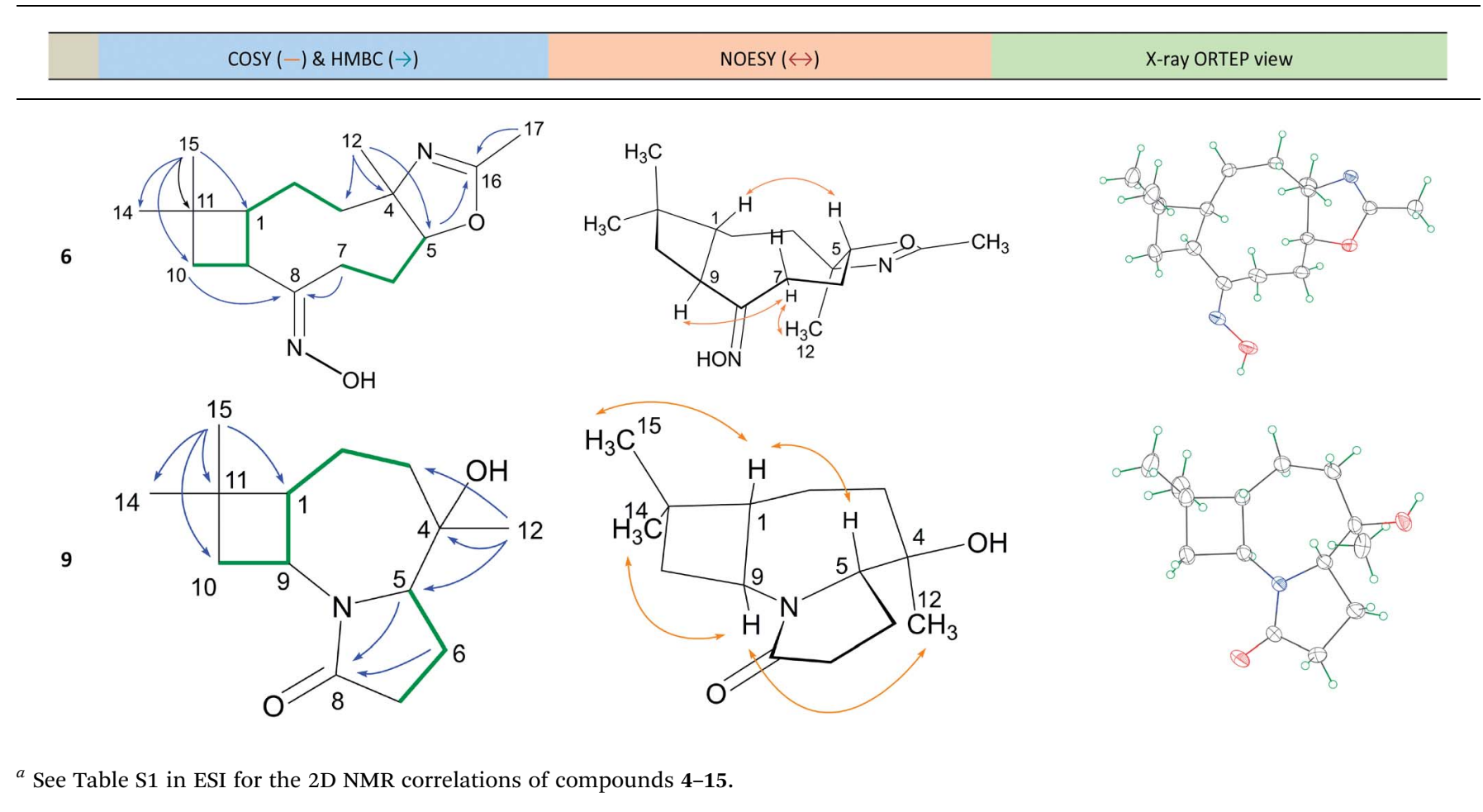

These findings showed that the $\mathrm{LiBF}_{4}$ did not provide access to the targeted remodeling skeletons. Pleasingly, we successfully formed the 2-oxazoline moiety at milder condition than that previously reported. ${ }^{21,22}$ To fully exploit build-in reactivity and increase rigid polycyclic molecules, Beckmann rearrangements of compound 3 were investigated with catalyst $p$-TsCl (Scheme 3). Compound 3 gave entirely different cyclization products 9-11, with polycyclic lactam skeletons. To acquire similar rearranged derivatives, the epoxyethane moiety in the starting material 3 was reduced into an endocyclic olefin. A following Beckmann rearrangement afforded a macrolactam 14 and two other polycyclic products 10 and 15.

To verify the skeleton remodeling, the structures of products 9-11, 14 and 15 were investigated by HRMS, IR, 1D and 2D
NMR. All the detailed correlations in 2D NMR were summarized in the Table $\mathrm{S} 1$ in ESI material. $\dagger$ For example, the MF of compound 9, $\mathrm{C}_{14} \mathrm{H}_{23} \mathrm{NO}_{2}$, was determined by HRESIMS. Two isolated spin systems were recognized by the COSY correlations as shown in Table 1 . The HMBC correlations of $\mathrm{H}-5$ and $\mathrm{H}_{2}-6$ to C-8 revealed cascaded Beckman-transannular remodeling on the caryophyllene's macrocycle. Furthermore, the X-ray analysis using $\mathrm{Cu} \mathrm{K} \alpha$ diffraction disclosed the N-containing 4/7/5 scaffold 9. The configuration was determined by NOESY correlations. The $\mathrm{H}-1(S)$ was selected as the reference group whose absolute configuration kept unchanged in the reaction process. The NOESY correlations among $\mathrm{H}-1, \mathrm{H}_{3}-15$ and $\mathrm{H}-5$ indicated that the newly formed chiral centre C-5 is $S$-configuration. The NOESY correlations from $\mathrm{H}-9$ to $\mathrm{H}_{3}-14$ and $\mathrm{H}_{3}-12$ indicated that

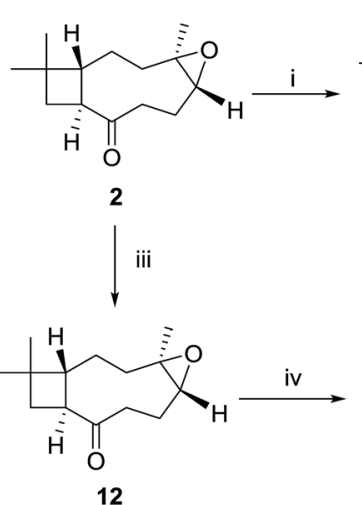<smiles>CC#CC1O[C@]1(C)CC[C@@H]1[C@H](C(=N)O)CC1(C)C</smiles><smiles>C[C@H]1[C@@H]2CCC(=O)N2[C@H]2CC[C@H]1C(C)(C)C2</smiles><smiles>CC1(C)C[C@@H]2[C@@H]1CC[C@]1(C)[C@H](O)CCC(=O)N21</smiles><smiles>CC1(C)C[C@H]2C(=O)N3CC[C@@H]4C(O)[C@]21CC[C@H]43</smiles>

9<smiles>CC1=CCC[C@@H]2C(CNO)[C@@H]1CC2(C)C</smiles>
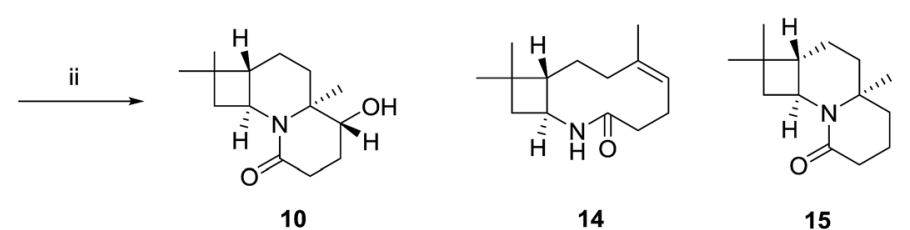

Scheme 3 Beckmann-transannular remodelling. (i) $\mathrm{NH}_{2} \mathrm{OH} \cdot \mathrm{HCl}, \mathrm{NaOH}$; (ii) $p-\mathrm{TsCl}_{1} \mathrm{Et}_{3} \mathrm{~N}, \mathrm{DMAP}$ (cat.), DCM, $3 \mathrm{~h}$, reflux; (iii) $\mathrm{Zn}$ powder; (iv) $\mathrm{NH}_{2} \mathrm{OH} \cdot \mathrm{HCl}, \mathrm{NaOH}$. 


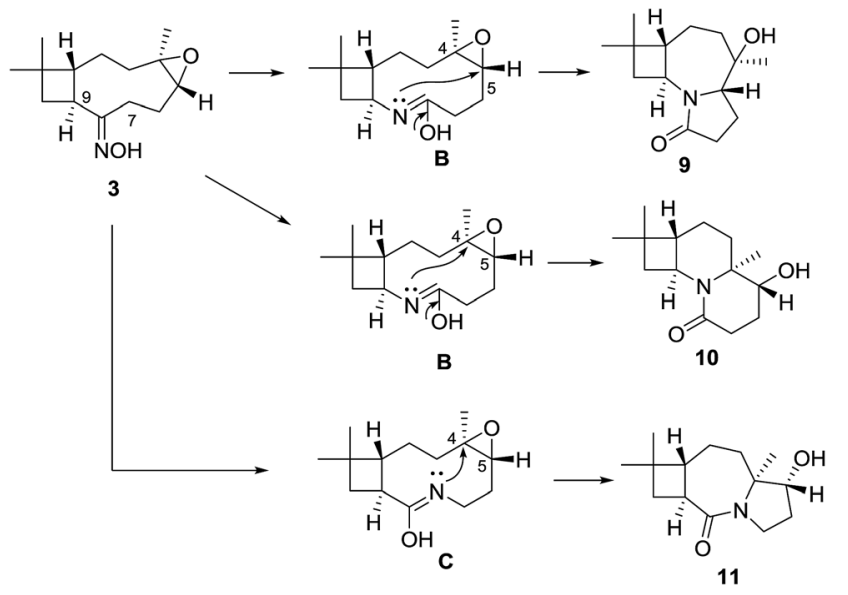

Scheme 4 Proposed mechanism for the Beckmann-transannular rearrangements to 9-11.

the chiral C-4 is $R$-configuration. The whole configuration was also confirmed by the X-ray diffraction as shown in Table 1 .

Based on the observed stereochemistry, a proposed mechanism for the formation of 9-11 is shown in Scheme 4. In the Beckmann process, the departure of the leaving group, TsO-, was accompanied by the $[1,2]$-shift of the C-7 or C-8 to afford the lactam tautomers $\mathbf{B}$ or $\mathbf{C}$. In the next step, transannular rearrangements occurred between the imines in $\mathbf{B}$ or $\mathbf{C}$ with the epo C-4 or C-5 to form the polycyclic skeletons of 9-11. Their structures indicated a cascaded Beckmann-transannular rearrangement in the process.

To evaluate the biological relevancy of product library, butyrylcholinesterase (BChE) was selected as bioassay target. Previous relationship observed between cholinergic dysfunction and Alzheimer's disease (AD) severity provides a rationale for the therapeutic use of cholinesterase inhibitors (ChEIs). ${ }^{23}$ Administration of ChEIs to increase the amount of cholines available for neural transmission has proven successful in relieving some cognitive as well as behavioral symptoms of AD. ${ }^{24,25}$ BChE inhibition was found to elevate brain acetylcholine, augment learning and reduce Alzheimer $\beta$-amyloid peptide. ${ }^{26}$ The bioassay indicated a weak inhibition of compound 15 ( $\left.\mathrm{IC}_{50} 134.6 \mu \mathrm{M}\right)$, with positive control, galanthamine $\left(\mathrm{IC}_{50} 82.6 \mu \mathrm{M}\right)$.

\section{Conclusions}

In conclusion, highly functionalized epoxy-macrocyclic sesquiterpenes, such as $\beta$-caryophyllene epoxide, endowed with a potential to generate rigid polycyclic structures not easily accessed by other methods. Our findings demonstrated that a Beckmann-transannular rearrangement on such a macrocycle is a concise approach to generate NP-like library with a lactam unit. In addition, $\beta$-caryophyllene and its epoxide are readily plentiful in natural source. Thus they can be served as ideal staring points to construct complex cyclic scaffolds covering unknown chemical space or bioactive space. On the basis of the present findings, combination of macrocyles with other naturally occurring bioactive blocker, will yield highly diverse products with NP-like scaffolds.

\section{Experimental procedures}

\section{General procedures}

UV spectra were obtained using an Evolution 300 UV-vis Spectrophotometer (Thermo Fisher Scientific Inc., USA). IR were recorded on a Bruker Tensor 27 spectrophotometer with $\mathrm{KBr}$ disks (Bruker Corp., German). Optical rotations were measured on an Autopol III automatic polarimeter (Rudolph Research Analytical, USA). ECD spectra were obtained on a Chirascan CD Spectrometer (Applied Photophysics Ltd., United Kingdom). ESI-MS were performed on an LTQ Fleet instrument (Thermo Fisher Scientific Inc., USA). HR ESIMS data were obtained by an AB Sciex Triple TOF 4600 system (AB Sciex Pte. Ltd., USA). 1D and 2D NMR spectra were recorded on an AVANCE III (500 $\mathrm{MHz}$ ) instrument (Bruker Corp., Germany). Chemical shifts were reported using solvent residual as the internal standard. Crystallographic data collection was carried out on a Bruker Smart Apex CCD area detector diffractometer with graphitemonochromated $\mathrm{Cu} \mathrm{K} \alpha$ radiation $(\lambda=1.54184 \AA)$ at $293(2) \mathrm{K}$ using $\omega$-scan technique. The diffraction data were integrated by using the SAINT program, which was also used for the intensity corrections for the Lorentz and polarization effects. Semiempirical absorption corrections were applied using SADABS program. Analytic and semi-preparative HPLC were performed on a Waters 1525 instrument (Waters Corp., USA). Column chromatography was performed on silica gel $(90-150 \mu \mathrm{m}$; Qingdao Marine Chemical Inc., China), Sephadex LH-20 (40-70 $\mu \mathrm{m}$; Amersham Pharmacia Biotech AB, Sweden), and Chromatorex $\mathrm{C}_{18}$ gel (40-75 $\mu \mathrm{m}$; Fuji Silysia Chemical LTD., JPN). $\mathrm{GF}_{254}$ plates (Qingdao Marine Chemical Inc., China) were used for thin-layer chromatography (TLC).

\section{Synthesis of oxime 3}

(-)- $\beta$-Caryophyllene epoxide ( $5.0 \mathrm{~g}, 22.7 \mathrm{mmol}), \mathrm{NaIO}_{4}(36.4 \mathrm{~g}$, $170.25 \mathrm{mmol}, 7.5 \mathrm{eq})$ was added to a mixed solvent $(25 \mathrm{ml}$ MeCN, $25 \mathrm{ml}$ EtOAc and $\left.37.5 \mathrm{H}_{2} \mathrm{O}\right)$ and $\mathrm{RuCl}_{3}(2.2 \% \mathrm{~mol})$ in a $250 \mathrm{ml}$ round-bottom flask equipped with a stir bar. After being stirred for $6 \mathrm{~h}$, the reaction mixture was filtered. The filtrate was extracted with EtOAc $(100 \mathrm{ml}, 3$ times). The combined organic layers were dried over $\mathrm{Na}_{2} \mathrm{SO}_{4}$ and concentrated under reduced pressure. The residue was purified on column chromatography (silica gel, petroleum ether $:$ EtOAc $=$ $10: 1, \mathrm{v} / \mathrm{v})$ to give the product $2(3.1 \mathrm{~g}, 61 \%$ yield $)$.

A solution of $2(1.11 \mathrm{~g}, 5 \mathrm{mmol}$ in $15 \mathrm{ml} \mathrm{EtOH})$ was added to a stirred solution of $\mathrm{NH}_{2} \mathrm{OH} \mathrm{HCl}(2.08 \mathrm{~g}, 30 \mathrm{mmol})$ in $15 \mathrm{ml} 2 \mathrm{~N}$ aqueous $\mathrm{NaOH}$. After being stirred for $30 \mathrm{~min}$ at room temperature, $60 \mathrm{ml} \mathrm{H}_{2} \mathrm{O}$ was added. Then the reaction mixture was extracted with DCM (100 ml, 3 times). The combined organic layers were dried over $\mathrm{Na}_{2} \mathrm{SO}_{4}$ and concentrated under reduced pressure to afford the crude residue. A further purification on column chromatography (silica gel, petroleum-EtOAc $20: 1, \mathrm{v} / \mathrm{v}$ ) to yield the product 3 (1.04 $\mathrm{g}, 87 \%$ yield). 
Compound 3. Colorless oil; $[\alpha]_{\mathrm{D}}^{30}-1.4\left(c\right.$ 0.23, $\left.\mathrm{CH}_{3} \mathrm{OH}\right)$; IR $(\mathrm{KBr}) \nu_{\max } 3315,2938,2864,1452,946,861 \mathrm{~cm}^{-1} ;{ }^{1} \mathrm{H}$ NMR $\left(\mathrm{CDCl}_{3}, 500 \mathrm{MHz}\right): \delta(\mathrm{ppm}) 1.74(\mathrm{dd}, J=9.3,2.4 \mathrm{~Hz}, \mathrm{H}-1,2 \mathrm{a})$, 1.65 (m, H-2b, 10b), 2.09 (dt, $J=12.8,3.5 \mathrm{~Hz}, \mathrm{H}-3 \mathrm{a}), 0.98$ (m, H3b); 2.62 (ddd, $J=12.2,7.5,4.3 \mathrm{~Hz}, \mathrm{H}-5), 2.33$ (m, H-6a), 1.45 (m, H-6b), 2.81 (q, $J=9.8 \mathrm{~Hz}, \mathrm{H}-7 \mathrm{a}), 2.42$ (m, H-7b), 2.86 (dd, $J=$ 11.0, 4.1 Hz, H-9), 1.95 (t, $J=9.9 \mathrm{~Hz}, \mathrm{H}-10 \mathrm{a}), 1.15$ (s, H $\left.\mathrm{H}_{3}-12\right), 1.02$ (s, $\left.\mathrm{H}_{3}-14\right), 1.03$ (s, $\left.\mathrm{H}_{3}-15\right) ;{ }^{13} \mathrm{C} \mathrm{NMR}\left(\mathrm{CDCl}_{3}, 125 \mathrm{MHz}\right): \delta(\mathrm{ppm})$ 49.4 (C-1), 23.1 (C-2), 37.7 (C-3), 59.8 (C-4), 64.2 (C-5), 26.6 (C-6), 38.4 (C-7), 162.3 (C-8), 45.9 (C-9), 23.7 (C-10), 35.2 (C-11), 16.0 (C12), 21.2 (C-14), 29.8 (C-15); ESIMS $m / z 238$ [M + H] $]^{+}$; HR ESIMS $m / z 238.1807[\mathrm{M}+\mathrm{H}]^{+}$(calcd for $\mathrm{C}_{14} \mathrm{H}_{24} \mathrm{NO}_{2} 238.1807$ ).

\section{DOS procedure catalysed by $\mathrm{LiBF}_{4}$}

$\mathrm{LiBF}_{4}(164.3 \mathrm{mg}, 1.75 \mathrm{mmol})$ was added slowly to a stirred solution of $3(1.04 \mathrm{~g}, 4.38 \mathrm{mmol})$ in $10 \mathrm{ml} \mathrm{MeCN}$. The reaction mixture was stirred at $45^{\circ} \mathrm{C}$ for $20 \mathrm{~h}$. The reaction was quenched with water $(30 \mathrm{ml})$. And the aqueous phase was extracted with DCM for 3 times. The combined organic layers were dried over $\mathrm{Na}_{2} \mathrm{SO}_{4}$, and concentrated under reduced pressure to give a crude product $(0.717 \mathrm{~g})$.

The crude was chromatographed on a silica gel column being eluted with $\mathrm{CHCl}_{3}-\mathrm{MeOH}$ (from $100: 1$ to $1: 1$ ) to give three fractions (Fr.1-Fr.3). Fr.1 (110 mg) was separated on an HPLC $\mathrm{C}_{18}$ column (Thermo BDS Hypersil $250 \times 10 \mathrm{~mm}$ ) with $80 \%$ methanol to yield compounds 7 (15 mg) and 8 (14 mg). Fr.2 (330 mg) was separated by a silica gel column chromatography $\left(\mathrm{CHCl}_{3}-\mathrm{MOH} 100: 1\right)$ to afford compounds 4 (200 mg). Fr.3 (260 $\mathrm{mg}$ ) was isolated on an HPLC $\mathrm{C}_{18}$ column with elution of $60 \%$ $\mathrm{MeOH}$ to yield $5(160 \mathrm{mg})$ and $6(40 \mathrm{mg})$.

Compound 4. Colorless oil; $[\alpha]_{\mathrm{D}}^{30}-85.1\left(c\right.$ 0.18, $\left.\mathrm{CH}_{3} \mathrm{OH}\right)$; IR $(\mathrm{KBr}) \nu_{\max } 3391,2942,1641,1435,1378,1059 \mathrm{~cm}^{-1} ;{ }^{1} \mathrm{H}$ NMR $\left(\mathrm{CDCl}_{3}, 500 \mathrm{MHz}\right): \delta(\mathrm{ppm}) 2.10(\mathrm{td}, J=10.9,5.4 \mathrm{~Hz}, \mathrm{H}-1), 1.79$ (m, H-2a), 1.41 (m, H-2b), 2.01 (d, $J=6.0 \mathrm{~Hz}, \mathrm{H}-3 \mathrm{a}$ ), 1.91 (m, H3b), 3.98 (dd, $J=7.3,3.5 \mathrm{~Hz}, \mathrm{H}-5), 3.95$ (dd, $J=7.1,3.0 \mathrm{~Hz}, \mathrm{H}-5$ ), 2.21 (m, H-6a), 1.63 (m, H-6b), 3.05 (dt, $J=13.7,5.4 \mathrm{~Hz}, \mathrm{H}-7 \mathrm{a}$ ), 1.98 (m, H-7b), 2.54 (td, $J=9.9,7.9 \mathrm{~Hz}, \mathrm{H}-9), 2.05$ (m, H-10a), 1.60 (d, $J=7.6 \mathrm{~Hz}, \mathrm{H}-10 \mathrm{~b}), 1.32$ (s, $\mathrm{H}_{3}-12$ ), 1.37 (s, H $\left.\mathrm{H}_{3}-12\right), 1.00$ (s, $\left.\mathrm{H}_{3}-14\right), 1.06$ (s, $\left.\mathrm{H}_{3}-15\right) ;{ }^{13} \mathrm{C} \mathrm{NMR}\left(\mathrm{CDCl}_{3}, 125 \mathrm{MHz}\right): \delta(\mathrm{ppm})$ 52.1 (C-1), 21.6 (C-2), 21.5 (C-2), 36.0 (C-3), 35.8 (C-3), 100.0 (C4), 101.3 (C-4), 73.2 (C-5), 73.1 (C-5), 28.1 (C-6), 28.0 (C-6), 26.4 (C-7), 162.2 (C-8), 40.5 (C-9), 33.9 (C-10), 34.7 (C-11), 20.0 (C-12), 19.8 (C-12) 22.2 (C-14) 30.1 (C-15); ESIMS m/z $258[\mathrm{M}+\mathrm{H}]^{+} ; 280$ $[\mathrm{M}+\mathrm{Na}]^{+}$; HR ESIMS $m / z$ 258.1867 $[\mathrm{M}+\mathrm{H}]^{+}$(calcd for $\mathrm{C}_{14} \mathrm{H}_{25} \mathrm{FNO}_{2}$ 258.1869).

Compound 5. Colorless needle, mp 260.8-261.1; $[\alpha]_{\mathrm{D}}^{30}-105.26$ (c 0.13, $\left.\mathrm{CH}_{3} \mathrm{OH}\right)$; IR (KBr) $\nu_{\max } 3378,2946,1640$, 1453, $1021 \mathrm{~cm}^{-1} ;{ }^{1} \mathrm{H}$ NMR (500 MHz, $\left.\mathrm{CDCl}_{3}\right) \delta \mathrm{ppm} 2.32\left(\mathrm{~m}, \mathrm{H}^{-}\right.$ 1, 2a), 1.93 (m, H-2b), 5.46 (dd, $J=4.9,3.9 \mathrm{~Hz}, \mathrm{H}-3), 4.57$ (dd, $J=$ 11.5, 3.9 Hz, H-5), 2.14 (m, H-6a), 2.01 (m, H-6b, 7b), 2.70 (ddd, $J$ = 11.7, 6.9, 4.1 Hz, H-7a), 2.449 (m, H-9), 1.81 (t, $J=10.4 \mathrm{~Hz}, \mathrm{H}-$ 10a), 1.61 (dd, $J=10.4,8.2 \mathrm{~Hz}, \mathrm{H}-10 \mathrm{~b}$ ), 1.67 (s, $\mathrm{H}_{3}-12$ ), 1.00 (s, $\left.\mathrm{H}_{3}-14\right), 1.04$ (s, $\left.\mathrm{H}_{3}-15\right) ;{ }^{13} \mathrm{C} \mathrm{NMR}\left(125 \mathrm{MHz}, \mathrm{CDCl}_{3}\right) \delta \mathrm{ppm} 51.2$ (C-1), 25.6 (C-2), 125.2 (C-3), 137.8 (C-4), 70.1 (C-5), 29.9 (C-6), 25.3 (C-7), 163.0 (C-8), 41.0 (C-9), 35.1 (C-10), 35.1 (C-11), 16.5
(C-12), 21.9 (C-14), 30.0 (C-15); ESIMS m/z $238[\mathrm{M}+\mathrm{H}]^{+} ; \mathrm{HR}$ ESIMS $m / z$ 238.1807 [M $+\mathrm{H}]^{+}$(calcd for $\mathrm{C}_{14} \mathrm{H}_{24} \mathrm{NO}_{2} 238.1807$ ).

Compound 6. Colorless needle, mp 191.2-191.8 ${ }^{\circ} \mathrm{C}$; $[\alpha]_{\mathrm{D}}^{30}-5.1\left(c 0.09, \mathrm{CH}_{3} \mathrm{OH}\right)$; IR (KBr) $\nu_{\max } 3436,2929,1644,1457$, $1392 \mathrm{~cm}^{-1} ;{ }^{1} \mathrm{H}$ NMR (500 MHz, $\left.\mathrm{CDCl}_{3}\right) \delta \mathrm{ppm} 1.80(\mathrm{dd}, J=9.6$, $3.2 \mathrm{~Hz}, \mathrm{H}-1$ ), 1.67 (m, H-2a), 1.47 (m, H-2b), 1.96 (m, H-3a), 1.61 (d, $J=7.9 \mathrm{~Hz}, \mathrm{H}-3 \mathrm{~b}), 4.166$ (dd, $J=10.2,3.6 \mathrm{~Hz}, \mathrm{H}-5), 2.39$ (m, H6a), 1.83 (dd, $J=8.4,2.0 \mathrm{~Hz}, \mathrm{H}-6 \mathrm{~b}), 3.12$ (dt, $J=13.6,5.7 \mathrm{~Hz}, \mathrm{H}-$ 7a), 1.87 (m, H-7b), 2.56 (td, $J=9.6,7.9 \mathrm{~Hz}, \mathrm{H}-9), 2.03(\mathrm{t}, J=$ $10.6 \mathrm{~Hz}, \mathrm{H}-10 \mathrm{a}), 1.58$ (d, $J=7.9 \mathrm{~Hz}, \mathrm{H}-10 \mathrm{~b}), 1.19$ (s, H-12), 0.99 (s, $\left.\mathrm{H}_{3}-14, \mathrm{H}_{3}-15\right), 1.91$ (s, $\left.\mathrm{H}_{3}-17\right) ;{ }^{13} \mathrm{C} \mathrm{NMR}\left(125 \mathrm{MHz}, \mathrm{CDCl}_{3}\right.$ ) $\delta$ ppm 54.3 (C-1), 24.6 (C-2), 42.5 (C-3), 70.8 (C-4), 85.2 (C-5), 25.4 (C-6), 26.7 (C-7), 161.1 (C-8), 44.3 (C-9), 34.4 (C-10), 34.9 (C-11), 20.1 (C-12), 21.5 (C-14), 29.7 (C-15), 162.4 (C-16), 14.0 (C-17); ESIMS $m / z 279[\mathrm{M}+\mathrm{H}]^{+} ;$HR ESIMS $m / z 279.2055[\mathrm{M}+\mathrm{H}]^{+}$ (calcd for $\mathrm{C}_{16} \mathrm{H}_{27} \mathrm{~N}_{2} \mathrm{O}_{2}$ 279.2072).

Compound 7. Colorless oil; $[\alpha]_{\mathrm{D}}^{30}+52.0\left(c 0.15, \mathrm{CH}_{3} \mathrm{OH}\right)$; IR $(\mathrm{KBr}) \nu_{\max } 3386,2932,1697,1450,943 \mathrm{~cm}^{-1} ;{ }^{1} \mathrm{H} \mathrm{NMR}(500 \mathrm{MHz}$, $\left.\mathrm{CDCl}_{3}\right) \delta$ ppm 1.81 (m, H-1), 1.42 (m, $\left.\mathrm{H}_{2}-2\right), 1.73$ (m, H-3a), 1.45 (m, H-3b), 2.55 (m, H-4), 2.63 (m, H-6a), 2.51 (m, H-6b), 2.84 (ddd, $J=18.3,8.2,1.8 \mathrm{~Hz}, \mathrm{H}-7 \mathrm{a}$ ), 2.63 (d, $J=9.5 \mathrm{~Hz}, \mathrm{H}-7 \mathrm{~b}), 2.48$ (m, H-9), 1.75 (m, H-10a), 1.69 (m, H-10b), 1.06 (d, $J=6.9 \mathrm{~Hz}$, $\left.\mathrm{H}_{3}-12\right), 0.96$ (s, $\left.\mathrm{H}_{3}-14\right), 1.00\left(\mathrm{~s}, \mathrm{H}_{3}-15\right) ;{ }^{13} \mathrm{C}$ NMR (125 MHz, $\left.\mathrm{CDCl}_{3}\right) \delta$ ppm 50.1 (C-1), 26.8 (C-2), 30.3 (C-3), 47.6 (C-4), 217.7 (C-5), 35.9 (C-6), 25.2 (C-7), 163.6 (C-8), 41.9 (C-9), 37.1 (C-10), 34.7 (C-11), 18.0 (C-12), 21.4 (C-14), 29.6 (C-15); ESIMS m/z $260.23[\mathrm{M}+\mathrm{Na}]^{+}$; HR ESIMS $m / z 238.1807[\mathrm{M}+\mathrm{H}]^{+}\left(\mathrm{C}_{14} \mathrm{H}_{23} \mathrm{NO}_{2}\right.$, calcd for $[\mathrm{M}+\mathrm{H}]^{+}$238.1807).

Compound 8. Colorless oil; $[\alpha]_{\mathrm{D}}^{30} 43.4$ ( $c$ 0.14, $\left.\mathrm{CH}_{3} \mathrm{OH}\right)$; IR (KBr) $\nu_{\max } 3373,2935,2865,1699,1451,945 \mathrm{~cm}^{-1} ;{ }^{1} \mathrm{H}$ NMR $\left(500 \mathrm{MHz}, \mathrm{CDCl}_{3}\right) \delta$ ppm 2.01 (m, H-1), 1.46 (m, H-2a), 1.40 (dt, $J=10.0,4.5 \mathrm{~Hz}, \mathrm{H}-2 \mathrm{~b}), 1.57$ (m, H-3a), 1.00 (m, overlapped, H3b), 2.40 (m, H-4), 2.71 (m, H-6a), 2.57 (dd, $J=12.1,10.6 \mathrm{~Hz}, \mathrm{H}-$ 6b), 2.70 (m, H-7a), 2.30 (m, H-7b), 3.26 (q, $J=9.5 \mathrm{~Hz}, \mathrm{H}-9$ ), 1.94 (dd, $\left.J=10.7,9.5 \mathrm{~Hz}, \mathrm{H}_{2}-10\right), 1.06$ (d, $J=6.9 \mathrm{~Hz}, \mathrm{H}_{3}-12$ ), 0.99 (s, $\mathrm{H}_{3}-14$ ), 1.00 (s, $\left.\mathrm{H}_{3}-15\right) ;{ }^{13} \mathrm{C} \mathrm{NMR}$ (125 MHz, $\mathrm{CDCl}_{3}$ ) $\delta$ ppm 43.9 (C-1), 28.3 (C-2), 29.3 (C-3), 50.7 (C-4), 217.6 (C-5), 29.3 (C-6), 35.4 (C-7), 164.5 (C-8), 32.6 (C-9), 37.2 (C-10), 35.5 (C-11), 18.0 (C-12), 22.1 (C-14), 30.0 (C-15); ESIMS m/z $260[\mathrm{M}+$ $\mathrm{Na}]^{+}$; HR ESIMS $m / z 238.1809[\mathrm{M}+\mathrm{H}]^{+}$(calcd for $\mathrm{C}_{14} \mathrm{H}_{24} \mathrm{NO}_{2}$ 238.1807).

\section{Beckmann remodeling of compound 3}

To a solution of compound $3(1.00 \mathrm{~g}, 4.2 \mathrm{mmol})$ in $10 \mathrm{ml}$ DCM, a solution of paratoluenesulfonyl chloride (p-TsCl, $2.00 \mathrm{~g}, 10.54$ $\mathrm{mmol}), \mathrm{Et}_{3} \mathrm{~N}$ (1.5 ml, $\left.10.54 \mathrm{mmol}\right)$ and DMAP (10.3 mg, $\left.2 \mathrm{~mol} \%\right)$ in DCM $(20 \mathrm{ml})$ was added dropwise. After being stirred for $3 \mathrm{~h}$ at room temperature, the reaction mixture was diluted with $20 \mathrm{ml} \mathrm{DCM}$ and washed with water $(2 \times 100 \mathrm{ml})$ and brine $(2 \times$ $100 \mathrm{ml}$ ). After being concentrated in vacuum and dried over $\mathrm{Na}_{2} \mathrm{SO}_{4}$, the crude product ( $820 \mathrm{mg}$ ) was yielded.

The crude product $(820 \mathrm{mg}$ ) was run on a silica gel column (gradient petroleum-EtOAc from $20: 1$ to $1: 2$ ) and a semipreparative HPLC $\mathrm{C}_{18}$ column (Thermo BDS Hypersil $250 \times 10$ $\mathrm{mm}, 60 \%$ methanol) to yield compounds $\mathbf{9}(80 \mathrm{mg}), \mathbf{1 0}$ (30 mg) and 11 (8 mg). 
Compound 9. Colorless needle, $\mathrm{mp}$ 222.8-223.6 ${ }^{\circ} \mathrm{C}$; $[\alpha]_{\mathrm{D}}^{30}+112.9\left(c 0.19, \mathrm{CH}_{3} \mathrm{OH}\right)$; IR $(\mathrm{KBr}) \nu_{\max } 3440,2978,2935$, 2866, 1657, 1447, 1291, $1252 \mathrm{~cm}^{-1} ;{ }^{1} \mathrm{H}$ NMR (500 MHz, $\mathrm{CDCl}_{3}$ ) $\delta$ ppm $1.74(\mathrm{dt}, J=12.3,1.8 \mathrm{~Hz}, \mathrm{H}-1), 1.63(\mathrm{~m}, \mathrm{H}-2 \mathrm{a}), 1.31(\mathrm{dt}, J=$ 12.3, $4.4 \mathrm{~Hz}, \mathrm{H}-2 \mathrm{~b}), 1.90$ (m, H-3a), 1.55 (m, H-3b), 3.90 (t, $J=$ $7.6 \mathrm{~Hz}, \mathrm{H}-5), 2.05$ (m, $\mathrm{H}_{2}-6$ ), 2.38 (ddd, $J=17.0,10.1,0.9 \mathrm{~Hz}, \mathrm{H}-$ 7a), 2.29 (m, H-7b), 3.42 (ddd, $J=16.7,6.9,1.8 \mathrm{~Hz}, \mathrm{H}-9), 2.33$ (m, H-10a), 1.59 (m, H-10b), 1.08 (s, $\mathrm{H}_{3}-12$ ), 1.01 (s, $\mathrm{H}_{3}-14$ ), 1.05 (s, $\left.\mathrm{H}_{3}-15\right) ;{ }^{13} \mathrm{C}$ NMR (125 MHz, $\mathrm{CDCl}_{3}$ ) $\delta \mathrm{ppm} 48.7$ (C-1), 24.4 (C-2), 45.0 (C-3), 75.1 (C-4), 66.4 (C-5), 20.4 (C-6), 30.7 (C-7), 174.8 (C8), 51.7 (C-9), 40.9 (C-10), 34.2 (C-11), 20.3 (C-12), 20.9 (C-14), 30.2 (C-15); ESIMS $m / z 238[\mathrm{M}+\mathrm{H}]^{+} ; 260[\mathrm{M}+\mathrm{Na}]^{+}$; HR ESIMS $m / z 238.1812[\mathrm{M}+\mathrm{H}]^{+}$(calcd for $\mathrm{C}_{14} \mathrm{H}_{24} \mathrm{NO}_{2} 238.1807$ ).

Compound 10. Colorless oil; $[\alpha]_{\mathrm{D}}^{30}+51.8\left(c 0.15, \mathrm{CH}_{3} \mathrm{OH}\right)$; IR $(\mathrm{KBr}) \nu_{\max } 3459,2080,1638 \mathrm{~cm}^{-1} ;{ }^{1} \mathrm{H}$ NMR (500 $\mathrm{MHz}, \mathrm{CDCl}_{3}$ ) $\delta$ ppm $1.41(\mathrm{dd}, J=11.3,3.2 \mathrm{~Hz}, \mathrm{H}-1), 1.53(\mathrm{dd}, J=11.0,3.2 \mathrm{~Hz}$, H-2a), 1.59 (m, H-2b), 1.44 (dd, $J=9.9,3.6 \mathrm{~Hz}, \mathrm{H}-3 \mathrm{a}$ ), 2.05 (dt, $J$ = 11.0, 3.2 Hz, H-3b), 3.73 (t, $J=8.1 \mathrm{~Hz}, \mathrm{H}-5), 1.89\left(\mathrm{~m}, \mathrm{H}_{2}-6\right)$, $2.40(\mathrm{t}, J=4.3 \mathrm{~Hz}, \mathrm{H}-7 \mathrm{a}), 2.43$ (ddd, $J=18.0,10.1,7.6 \mathrm{~Hz}, \mathrm{H}-7 \mathrm{~b})$, $3.14(\mathrm{td}, J=10.7,7.3 \mathrm{~Hz}, \mathrm{H}-9), 2.09$ (t, $J=10.4,6.9 \mathrm{~Hz}, \mathrm{H}-10 \mathrm{a})$, 2.32 (dd, $J=10.4,6.9 \mathrm{~Hz}, \mathrm{H}-10 \mathrm{~b}), 1.27$ (s, $\mathrm{H}_{3}-12$ ), 1.10 (s, $\mathrm{H}_{3}-14$, 15); ${ }^{13} \mathrm{C}$ NMR (125 MHz, $\mathrm{CDCl}_{3}$ ) $\delta$ ppm 52.9 (C-1), 22.3 (C-2), 37.2 (C-3), 61.4 (C-4), 74.5 (C-5), 25.7 (C-6), 30.3 (C-7), 169.0 (C-8), 49.4 (C-9), 43.3 (C-10), 37.1 (C-11), 17.2 (C-12), 20.6 (C-14), 30.3 (C-15); ESIMS $m / z 238[\mathrm{M}+\mathrm{H}]^{+}, 260[\mathrm{M}+\mathrm{Na}]^{+}$; HR ESIMS $m / z 238.1813[\mathrm{M}+\mathrm{H}]^{+}$(calcd for $\mathrm{C}_{14} \mathrm{H}_{24} \mathrm{NO}_{2}, 238.1807$ ).

Compound 11. Colorless needle, mp 222.1-222.5 ${ }^{\circ} \mathrm{C}$; $[\alpha]_{\mathrm{D}}^{30}-47.4\left(c\right.$ 0.14, $\left.\mathrm{CH}_{3} \mathrm{OH}\right)$; IR (KBr) $\nu_{\max } 3453,2950,2080$, 1638, 1436, $1017 \mathrm{~cm}^{-1} ;{ }^{1} \mathrm{H}$ NMR (500 MHz, $\left.\mathrm{CDCl}_{3}\right) \delta \mathrm{ppm} 1.74$ (m, H-1), $1.62\left(\mathrm{~m}, \mathrm{H}_{2}-2\right), 2.12(\mathrm{dt}, J=14.2,3.5 \mathrm{~Hz}, \mathrm{H}-3 \mathrm{a}), 1.32(\mathrm{~m}$, H-3b), 3.85 (dd, $J=11.0,6.3 \mathrm{~Hz}, \mathrm{H}-5$ ), 2.07 (m, H-6a), 1.78 (td, $J$ $=9.3,1.9 \mathrm{~Hz}, \mathrm{H}-6 \mathrm{~b}), 3.69(\mathrm{dd}, J=9.3,2.5 \mathrm{~Hz}, \mathrm{H}-7 \mathrm{a}), 3.20(\mathrm{td}, J=$ 11.7, $7.3 \mathrm{~Hz}, \mathrm{H}-7 \mathrm{~b}), 2.82(\mathrm{td}, J=8.2,2.2 \mathrm{~Hz}, \mathrm{H}-9), 1.93(\mathrm{t}, J=$ 10.4 Hz, H-10a), 1.70 (m, H-10b), 1.22 (s, H $\left.\mathrm{H}_{3}-12\right), 1.08$ (s, $\mathrm{H}_{3}-14$ ), 1.04 (s, $\left.\mathrm{H}_{3}-15\right) ;{ }^{13} \mathrm{C}$ NMR (125 MHz, $\mathrm{CDCl}_{3}$ ) $\delta \mathrm{ppm} 49.6$ (C-1), 23.9 (C-2), 38.5 (C-3), 63.8 (C-4), 80.5 (C-5), 28.2 (C-6), 42.3 (C7), 175.7 (C-8), 39.8 (C-9), 35.0 (C-10), 35.1 (C-11), 16.7 (C-12), 21.4 (C-14), 29.5 (C-15); ESIMS $m / z 238[\mathrm{M}+\mathrm{H}]^{+} ; 260[\mathrm{M}+$ $\mathrm{Na}]^{+}$; HR ESIMS $m / z$ 238.1806 $[\mathrm{M}+\mathrm{H}]^{+}$(calcd for $\mathrm{C}_{14} \mathrm{H}_{24} \mathrm{NO}_{2}$ 238.1807).

\section{Synthesis of compound 13}

To a solution of compound $2(2.00 \mathrm{~g}, 9 \mathrm{mmol})$ in anhydrous EtOH $(90 \mathrm{ml})$, activated zinc powder $(100 \mathrm{~g})$ was added. The reaction mixture was refluxed for $48 \mathrm{~h}$. Then the reaction mixture was cooled to room temperature and filtered. The residue was washed with water, and then extracted with EtOAc $(3 \times 120 \mathrm{ml})$. The combined extraction were dried over $\mathrm{Na}_{2} \mathrm{SO}_{4}$ and concentrated under reduced pressure to afford crude product. The crude product was purified on a silica gel column (petroleum-EtOAc $60: 1)$ to yield the compound 12 (1.00 g, 54\% yield).

To a stirred solution of $\mathrm{NH}_{2} \mathrm{OH} \mathrm{HCl}(2.00 \mathrm{~g}, 28.8 \mathrm{mmol})$ in $14.5 \mathrm{ml}$ aqueous $\mathrm{NaOH}(2 \mathrm{M})$, a solution of compound 12 $(1.00 \mathrm{~g}, 4.8 \mathrm{mmol})$ in ethanol $(15 \mathrm{ml})$. After being stirred for $30 \mathrm{~min}$ at room temperature, $60 \mathrm{ml}$ water was added. The reaction mixture was extracted with DCM $(100 \mathrm{ml})$ for 3 times. The combined organic layers were dried over $\mathrm{Na}_{2} \mathrm{SO}_{4}$ and concentrated under reduced pressure to yield the crude product. The crude product was run on a silica gel column chromatography (petroleum-EtOAc $20: 1$ ) to afford compound 13 (960 mg, 90\% yield).

Compound 13. Colorless oil; $[\alpha]_{\mathrm{D}}^{30}+91.9\left(c 0.13, \mathrm{CH}_{3} \mathrm{OH}\right)$; IR $(\mathrm{KBr}) \nu_{\max } 3445,2939,1640,1451 \mathrm{~cm}^{-1} ;{ }^{1} \mathrm{H}$ NMR $(500 \mathrm{MHz}$, $\left.\mathrm{CDCl}_{3}\right) \delta$ ppm 1.89 (m, H-1), 1.56 (m, H-2a), 1.49 (m, H-2b), 2.10 (dt, $J=11.7,3.4 \mathrm{~Hz}, \mathrm{H}-3 \mathrm{a}), 1.93$ (m, H-3b), 5.39 (dd, $J=10.1$, 6.1 Hz, H-5), 2.58 (m, H-6a), 2.21 (td, $J=12.6,6.1 \mathrm{~Hz}, \mathrm{H}-6 \mathrm{~b}), 2.44$ (m, H-7a), 2.37 (m, H-7b), 2.53 (m, H-9), 1.82 (t, $J=10.6 \mathrm{~Hz}, \mathrm{H}-$ 10a), 1.68 (dd, $J=10.6,8.5 \mathrm{~Hz}, \mathrm{H}-10 \mathrm{~b}$ ), 1.61 (d, $J=0.9 \mathrm{~Hz}, \mathrm{H}_{3}-12$ ), 1.00 (s, $\left.\mathrm{H}_{3}-14\right), 1.04$ (s, $\left.\mathrm{H}_{3}-15\right) ;{ }^{13} \mathrm{C}$ NMR (125 MHz, $\left.\mathrm{CDCl}_{3}\right) \delta \mathrm{ppm}$ 52.2 (C-1), 28.9 (C-2), 39.6 (C-3), 136.3 (C-4), 125.1 (C-5), 22.4 (C6), 27.9 (C-7), 164.7 (C-8), 46.4 (C-9), 37.9 (C-10), 34.1 (C-11), 15.8 (C-12), 22.1 (C-14), 29.9 (C-15); ESIMS $m / z 222[\mathrm{M}+\mathrm{H}]^{+}$; HR ESIMS $m / z 222.1875[\mathrm{M}+\mathrm{H}]^{+}$(calcd for $\mathrm{C}_{14} \mathrm{H}_{24} \mathrm{NO} 222.1858$ ).

\section{Beckmann remodeling of 13}

To a $10 \mathrm{ml}$ DCM solution of compound 13 (0.95 g, $4.3 \mathrm{mmol})$, a $10 \mathrm{ml}$ DCM solution of $p$-TsCl $(2.10 \mathrm{~g}, 10.75 \mathrm{mmol}), \mathrm{Et}_{3} \mathrm{~N}$ $(1.5 \mathrm{ml}, 10.75 \mathrm{mmol})$ and DMAP $(10 \mathrm{mg})$ was added dropwise. After being stirred for $3 \mathrm{~h}$ at room temperature, the mixture was diluted with $20 \mathrm{ml}$ DCM and washed with water $(2 \times 100 \mathrm{ml})$ and brine $(2 \times 100 \mathrm{ml})$. The organic layer was dried over $\mathrm{Na}_{2} \mathrm{SO}_{4}$ and concentrated in vacuum to afford the crude product ( 820 $\mathrm{mg}$ ). Remaining reactants were removed on a silica gel column (petroleum-EtOAc $20: 1-1: 2$ ). A further HPLC $\mathrm{C}_{18}$ chromatography (Thermo BDS Hypersil $250 \times 10 \mathrm{~mm}, 60 \% \mathrm{MeOH}$ ) afforded compounds 10 (8 mg), 14 (90 mg) and 15 (16 mg).

Compound 14. Colorless oil; $[\alpha]_{\mathrm{D}}^{31}-11.4\left(c\right.$ 0.14, $\left.\mathrm{CH}_{3} \mathrm{OH}\right)$; IR (KBr) $\nu_{\max } 3308,2939,2860,1655,1548,1454,1336,1287,1186$ $\mathrm{cm}^{-1} ;{ }^{1} \mathrm{H}$ NMR $\left(500 \mathrm{MHz}, \mathrm{CDCl}_{3}\right) \delta \mathrm{ppm} 1.44(\mathrm{~m}, \mathrm{H}-1), 1.51(\mathrm{~m}, \mathrm{H}-$ 2a), 1.58 (dd, $J=3.0,1.9 \mathrm{~Hz}, \mathrm{H}-2 \mathrm{~b}), 1.88$ (td, $J=12.7,3.0 \mathrm{~Hz}, \mathrm{H}-$ 3a), 2.24 (d, $J=12.0 \mathrm{~Hz}, \mathrm{H}-3 \mathrm{~b}), 5.12$ (dt, $J=11.7,1.3 \mathrm{~Hz}, \mathrm{H}-5), 2.15$ (m, H-6a), 2.77 (qd, $J=11.7,5.4 \mathrm{~Hz}, \mathrm{H}-6 \mathrm{~b}), 1.94$ (ddd, $J=12.0$, 11.7, $5.8 \mathrm{~Hz}, \mathrm{H}-7 \mathrm{a}$ ), 2.36 (ddd, $J=12.0,5.4,2.0 \mathrm{~Hz}, \mathrm{H}-7 \mathrm{~b}$ ), 3.97 (dt, $J=18.0,8.9 \mathrm{~Hz}, \mathrm{H}-9), 1.47$ (m, H-10a), 2.04 (dd, $J=10.7,7.9 \mathrm{~Hz}$, $\mathrm{H}-10 \mathrm{~b}), 1.60$ (s, $\mathrm{H}_{3}-12$ ), 0.96 (s, $\left.\mathrm{H}_{3}-14\right), 1.02$ (s, $\left.\mathrm{H}_{3}-15\right), 4.96$ (d, $J=$ $8.9 \mathrm{~Hz}, \mathrm{HN}) ;{ }^{13} \mathrm{C} \mathrm{NMR}\left(125 \mathrm{MHz}, \mathrm{CDCl}_{3}\right) \delta \mathrm{ppm} 58.1(\mathrm{C}-1), 26.4(\mathrm{C}-$ 2), 41.0 (C-3), 138.9 (C-4), 122.7 (C-5), 26.6 (C-6), 38.2 (C-7), 174.3 (C-8), 47.9 (C-9), 42.1 (C-10), 31.3 (C-11), 15.3 (C-12), 21.9 (C-14), 29.9 (C-15); ESIMS $m / z 222[\mathrm{M}+\mathrm{H}]^{+} ; 244[\mathrm{M}+\mathrm{Na}]^{+}$; HR ESIMS $m / z 222.1853[\mathrm{M}+\mathrm{H}]^{+}$(calcd for $\mathrm{C}_{14} \mathrm{H}_{24} \mathrm{NO} 222.1858$ ).

Compound 15. Colorless oil; $[\alpha]_{\mathrm{D}}^{22}+65.9(c 0.18, \mathrm{MeOH}) ; \mathrm{IR}$ (KBr) $\nu_{\max } 2943,2871,1641,1451,1369,1154 \mathrm{~cm}^{-1}$; ${ }^{1} \mathrm{H}$ NMR $\left(500 \mathrm{MHz}, \mathrm{CDCl}_{3}\right) \delta$ ppm 1.43 (m, H-1), 1.53 (m, $\left.\mathrm{H}_{2}-2\right), 1.67$ (m, $\mathrm{H}_{2}-3$ ), 1.54 (m, H-6a), 1.66 (m, H-6b), 1.72 (m, H-7a), 1.83 (m, H7b), 2.30 (m, H $\left.\mathrm{H}_{2}-8\right), 3.10$ (dt, $\left.J=14.2,3.5 \mathrm{~Hz}, \mathrm{H}-9\right), 2.16$ (t, $J=$ $10.1 \mathrm{~Hz}, \mathrm{H}-10 \mathrm{a}$ ), 2.29 (m, H-10b), 1.29 (s, H $\mathrm{H}_{3}-12$ ), 1.08 (s, $\mathrm{H}_{3}-14$, 15); ${ }^{13} \mathrm{C}$ NMR (125 MHz, $\mathrm{CDCl}_{3}$ ) $\delta$ ppm 53.5 (C-1), 22.5 (C-2), 38.5 (C-3), 58.3 (C-4), 40.5 (C-5), 17.0 (C-6), 32.2 (C-7), 169.4 (C-8), 49.3 (C-9), 43.3 (C-10), 36.8 (C-11), 23.5 (C-12), 20.5 (C-14), 30.2 (C-15); ESIMS $m / z 222[\mathrm{M}+\mathrm{H}]^{+}$; HR ESIMS $m / z 222.1860$ $[\mathrm{M}+\mathrm{H}]^{+}$(calcd for $\mathrm{C}_{14} \mathrm{H}_{24} \mathrm{NO}, 222.1858$ ). 


\section{Bioassay}

Butyrylcholine esterase inhibitory activity was measured by spectrophotometric method reported previously. ${ }^{27}$ The reaction mixture contained $120 \mu \mathrm{l}$ PBS buffer (pH 7.4), $10 \mu$ l butyrylcholinesterase solution, $10 \mu \mathrm{l}$ of test compound solution, which were mixed and incubated for $10 \mathrm{~min}\left(37^{\circ} \mathrm{C}\right)$. The reaction was initiated by the addition of $30 \mu \mathrm{l} S$-butyrylthiocholine chloride and $30 \mu \mathrm{l}$ DTBN. The hydrolysis of butyrylthiocholine iodide was monitored at $412 \mathrm{~nm}$ after $30 \mathrm{~min}$. Galanthamine was used as positive control. All the reactions were performed in triplicate. The percentage inhibition was calculated as follows:

$$
\text { Inhibition }(\%)=\left(1-A_{\mathrm{i}}\right) \times 100 / A_{0}
$$

where $A_{0}$ is the activity of enzyme without test compound and $A_{\mathrm{i}}$ is the activity of enzyme with test compounds.

\section{Conflicts of interest}

There are no conflicts to declare.

\section{Acknowledgements}

This work is financially supported by State Key Laboratory of Medicinal Chemical Biology, Nankai University (No. 201502003) and the Natural Science and Basic Research Plan of Shaanxi Province (No. 2016JM2002).

\section{Notes and references}

1 T. Rodrigues, D. Reker, P. Schneider and G. Schneider, Nat. Chem., 2016, 8, 531.

2 J. M. Gao, W. J. Wu, J. W. Zhang and Y. Konishi, Nat. Prod. Rep., 2007, 24, 1153.

3 J. M. Gao, S. X. Yang and J. C. Qin, Chem. Rev., 2013, 113, 4755.

4 J. W.-H. Li and J. C. Vederas, Science, 2009, 325, 161.

5 K. C. Morrison and P. J. Hergenrother, Nat. Prod. Rep., 2014, 31, 6.

6 G. Prabhu, S. Agarwal, V. Sharma, S. M. Madurkar, P. Munshi, S. Singh and S. Sen, Eur. J. Med. Chem., 2015, 95, 41.

7 J. Kim, H. Kim and S. B. Park, J. Am. Chem. Soc., 2014, 136, 14629.
8 W. R. J. D. Galloway, A. Isidro-Llobet and D. R. Spring, Nat. Commun., 2010, 1, 80.

9 H. Kikuchi, T. Nishimura, E. Kwon, J. Kawai and Y. Oshima, Chem.-Eur. J., 2016, 22, 15819.

10 L. Huang, L.-X. Dai and S.-L. You, J. Am. Chem. Soc., 2016, 138, 5793.

11 K. Burger, B. Mühl, T. Harasim, M. Rohrmoser, A. Malamoussi, M. Orban, M. Kellner, A. Gruber-Eber, E. Kremmer, M. Hölzel and D. Eick, J. Biol. Chem., 2010, 285, 12416.

12 D.-H. Lai, Z.-D. Yang, W.-W. Xue, J. Sheng, Y. Shi and X.-J. Yao, Fitoterapia, 2013, 89, 257.

13 R. A. Ramli, W. Lie and S. G. Pyne, J. Nat. Prod., 2014, 77, 894.

14 W. Gao, A. P.-C. Chen, C.-H. Leung, E. A. Gullen, A. Fürstner, Q. Shi, L. Wei, K.-H. Lee and Y.-C. Cheng, Bioorg. Med. Chem. Lett., 2008, 18, 704.

15 E. Reyes, U. Uria, L. Carrillo and J. L. Vicario, Tetrahedron, 2014, 70, 9461.

16 B. R. Balthaser, M. C. Maloney, A. B. Beeler, J. A. Porco and J. K. Snyder, Nat. Chem., 2011, 3, 969.

17 M. Mewald, J. W. Medley and M. Movassaghi, Angew. Chem., Int. Ed., 2014, 53, 11634.

18 Q. Zhang, H.-Y. Tang, M. Chen, J. Yu, H. Li and J.-M. Gao, Org. Biomol. Chem., 2017, 15, 4456.

19 H.-Y. Tang, J.-M. Gao and Q. Zhang, RSC Adv., 2015, 5, 72433. 20 I. G. Collado, J. R. Hanson and A. J. Macías-Sánchez, Nat. Prod. Rep., 1998, 15, 187.

21 J. L. García Ruano and C. García Paredes, Tetrahedron Lett., 2000, 41, 5357.

22 M. Shimizu and H. Yoshioka, Heterocycles, 1988, 27, 2527.

23 J. C. Morris, Am. Fam. Physician, 2006, 74, 747.

24 S. Stepankova and K. Komers, Curr. Enzyme Inhib., 2008, 58, 160.

25 J. Grutzendler and J. C. Morris, Drugs, 2001, 61, 41.

26 N. H. Greig, T. Utsuki, D. K. Ingram, Y. Wang, G. Pepeu, C. Scali, Q.-S. Yu, J. Mamczarz, H. W. Holloway, T. Giordano, D. Chen, K. Furukawa, K. Sambamurti, A. Brossi and D. K. Lahiri, Proc. Natl. Acad. Sci. U. S. A., 2005, 102, 17213.

27 M. Danish, M. A. Raza, Sehrish and N. Anjum, Asian J. Chem., 2015, 27, 3925. 